\title{
THE COHOMOLOGY ALGEBRA OF CERTAIN LOOP SPACES
}

\section{EDWARD HALPERN}

The purpose of this paper ${ }^{1}$ is to determine the cohomology algebra of a loop space over a topological space whose cohomology algebra is a truncated polynomial algebra generated by an element of even degree. As special cases we obtain the well-known results when the base space has as cohomology algebra an exterior algebra (the base space an even dimensional sphere) or a polynomial algebra (the base space infinite dimensional complex projective space; compare also Theorem 2 in [1]). In particular, the result is applicable to loop spaces over complex and quaternionic projective $n$-spaces and the Cayley plane.

Throughout, $A$ will denote a commutative ring with unit and $A$ algebra will mean an associative $A$-algebra with unit.

1. Augmented spectral sequences of algebras. A differential $A$ module consists of an $A$-module $E$ and a (module) endomorphism $d: E \rightarrow E$ such that $d d=0$. The map $d$ is called a differential and the elements of its kernel and image are called cycles and boundaries respectively; the quotient module $H(E)=$ Kernel of $d /$ Image of $d$ is called the derived module. A differential A-algebra consists of an $A$ algebra which is a differential $A$-module and an automorphism $\omega: E \rightarrow E$ such that

$$
d \omega+\omega d=0, \quad d(x y)=(d x) y+\omega(x) d y, \quad x, y \in E .
$$

It follows that $H(E)$ has a naturally induced multiplication under which $H(E)$ is an $A$-algebra. An augmentation of a differential $A$ algebra is an algebra homomorphism $\alpha: E \rightarrow A$ with right inverse $\beta: A \rightarrow E$ such that $\alpha d=0$. It follows that $H(E)$ has a naturally induced augmentation $\bar{\alpha}$. The kernel of $\alpha$ will be denoted by $E^{+}$.

An augmented spectral sequence of $A$-algebras is a sequence of augmented differential $A$-algebras $\left(E_{r}\right), r \geqq 0$, such that $E_{r+1}=H\left(E_{r}\right)$ and $\alpha_{r+1}=\bar{\alpha}_{r}$. The limit of $\left(E_{r}\right)$ is the augmented $A$-algebra defined as follows: An element $x_{r} \in E_{r}$ is called a permanent cycle if it is a cycle and its successive projections in $E_{r+1}, E_{r+2}, \cdots$ are cycles. Let $E_{\infty}$ be the set of sequences $\left(x_{r}\right)$ where $x_{r}$ is a permanent cycle of $E_{r}$ and $x_{r+1}$ is the projection of $x_{r}$ in $E_{r+1}$, with two such sequences identified if $x_{r}=x_{r}^{\prime}$ for all $r \geqq r_{0}$. Defining

Received by the editors August 6, 1957.

${ }^{1}$ Appeared as a portion of the author's Doctoral Dissertation, University of Chicago, March 1957. 


$$
\begin{aligned}
& \left(x_{r}\right)+\left(y_{r}\right)=\left(x_{r}+y_{r}\right), \quad a\left(x_{r}\right)=\left(a x_{r}\right), \quad a \in A, \\
& \left(x_{r}\right)\left(y_{r}\right)=\left(x_{r} y_{r}\right), \quad \alpha\left(x_{r}\right)=\alpha_{r} x_{r},
\end{aligned}
$$

where $\alpha_{r}$ is the augmentation of $E_{r}$, makes $E_{\infty}$ into an augmented $A$-algebra. The augmentation $\alpha$ of $E_{\infty}$ is well-defined and its kernel $E_{\infty}^{+}$is the subalgebra defined by permanent cycles $\left(x_{r}\right)$ such that $x_{r} \in E_{r}^{+}$. The spectral sequence is acyclic if $E_{\infty}^{+}=0$.

An augmented spectral sequence of $A$-algebras is canonical if the sequence $\left(E_{r}\right)$ is defined for $r \geqq 2$ and for each $r$ :

(a) $E_{r}$ is a bigraded algebra, $E_{r}=\sum_{p, q} E_{r}^{p, q}$, with $E_{r}^{p, q}=0$ if $p<0$ or $q<0$; moreover, the multiplication in $E_{r}$ is anticommutative with respect to total degree $p+q$.

(b) The differential $d_{r}$ is bihomogeneous of bidegree $(r, 1-r)$.

(c) The automorphism $\omega_{r}$ is given by $\omega_{r}(x)=(-1)^{p+q} x$ for $x \in E_{r}^{p, q}$.

(d) The augmentation $\alpha_{r}$ maps $E_{r}^{0,0}$ isomorphically onto $A$.

(e) $E_{r+1}^{p, q}=H\left(E_{r}^{p, q}\right)$.

It follows from (d) that $E_{\tau}^{+}=\sum_{p+q>0} E_{r}^{p, q}$. From (e) it follows that $E_{\infty}$ has a naturally induced bigrading with $E_{\infty}^{p, q}=0$ if $p<0$ or $q<0$. In view of (d) it then follows that $\alpha$ maps $E_{\infty}^{0,0}$ isomorphically onto $A$, and $E_{\infty}^{+}=\sum_{p+q>0} E_{\infty}^{p, q}$. Thus acyclicity of the spectral sequence is equivalent to the statement that $E_{\infty}^{p, q}=0$ for $p+q>0$. It may be readily proved that

$$
E_{r}^{p, q}=E_{r+1}^{p, q}=\cdots=E_{\infty}^{p, q}
$$$$
\text { if } r>p \text { and } r>q+1 \text {. }
$$

The spectral sequence is said to be initially decomposable if

$$
E_{2}^{p, q}=E_{2}^{p, 0} \cdot E_{2}^{0, q}
$$

more precisely, if every $y \in E_{2}^{p, q}$ can be written as a sum of products $x z$ where $x \in E_{2}^{p, 0}$ and $z \in E_{2}^{0, q}$. Note that $B=\sum E_{2}^{p, 0}$ and $F=\sum E_{2}^{0, q}$ are graded subalgebras of $E_{2}$.

2. Monogenic twisted polynomial algebras. A monogenic twisted polynomial $A$-algebra of height $h, 2 \leqq h \leqq \infty$, and type $t=\left(t_{m, n}\right)$ is a free $A$-module generated by a sequence of elements $x_{0}, x_{1}, \cdots, x_{h-1}$ with multiplication defined by

$$
x_{m} x_{n}= \begin{cases}t_{m, n} x_{m+n} & m+n<h, \\ 0 & m+n \geqq h,\end{cases}
$$

where the $t_{m, n}$ are nonzero elements of $A$ which satisfy:

$$
\begin{aligned}
t_{0, n} & =1, \quad t_{m, 0}=1, \\
t_{m, n} & =t_{n, m},
\end{aligned}
$$




$$
t_{m, n} t_{m+n, k}=t_{m, n+k} t_{n, k} .
$$

From (2.3) and (2.4) follow commutativity and associativity respectively; from (2.1) and (2.2) follows that $x_{0}=1$. The powers $x_{1}^{m}$ are related to the generators $x_{m}$ as follows: Putting $t_{k}=t_{1, k-1},(k>0)$, then by induction one proves

$$
x_{1}^{m}=t_{1} t_{2} \cdots t_{m} x_{m} .
$$

We shall write $x_{1}=x$ and denote the algebra by $A[x, h, t]$. In particular, if $t_{m, n}=1$ for all $m+n<h$ then the algebra is the ordinary (truncated) polynomial algebra of height $h$ which we shall denote by $A[x, h]$; evidently $A[x, 2]$ is the exterior algebra $\Lambda_{A}(x)$. If each $t_{m, n}$ differs from the binomial coefficient $(m, n)=m+n ! / m ! n !$ by a unit then the algebra will be said to be of binomial type.

A monogenic twisted $A$-algebra of binomial type is a free $A$-module generated by a sequence of elements $\left(x_{0}, x_{1}, x_{2}, \cdots\right)$ with multiplication defined by

$$
x_{m} x_{n}=(m, n) x_{m+n} .
$$

It will be denoted by $T_{A}\left(x_{0}, x_{1}, x_{2}, \cdots\right)$. Since the binomial coefficients satisfy (2.2), (2.3), and (2.4), $T_{A}\left(x_{0}, x_{1}, x_{2}, \cdots\right)$ is associative, commutative, and $x_{0}=1$.

We note the following readily proved property of the binomial coefficients $(m, n)$ modulo a prime $p$ :

$$
(m, n)=\left(m_{0}, n_{0}\right)\left(m_{1}, n_{1}\right) \cdots\left(m_{j}, n_{j}\right),
$$

where

$$
\begin{array}{r}
m=m_{0}+m_{1} p+\cdots+m_{i} p^{i}, \quad n=n_{0}+n_{1} p+\cdots+n_{j} p^{j}, \\
i \leqq j,
\end{array}
$$

are the $p$-adic expansions of $m$ and $n$, and $m_{k}=0$ if $k>i$.

Proposition 1. (a) If $A$ has characteristic zero then

$$
T_{A}\left(x_{0}, x_{1}, x_{2}, \cdots\right)=A[x, \infty, t] .
$$

(b) If $A$ has characteristic prime $p$ then there is an algebra isomorphism ${ }^{2}$

(2.10) $\phi: T_{A}\left(x_{0}, x_{1}, x_{2}, \cdots\right) \cong \otimes_{i \geq 0} A\left[x_{p} i, p, t^{(i)}\right], \quad t_{m, n}^{(i)}=(m, n)$.

Proof. To prove (a) we need only note that $(m, n) \neq 0$. (b) For each $i \geqq 0$ define $p$ elements $y_{m}^{(i)}=x_{m p i}, 0 \leqq m<p$. If $0 \leqq m<p$ and $0 \leqq n<p$ then, using (2.6) and (2.7), we have

2 By $\otimes_{i \geq 0}$ is meant the "weak" tensor product. 


$$
y_{m}^{(i)} y_{n}^{(i)}=x_{m p^{i}} x_{n p^{i}}=\left(m p^{i}, n p^{i}\right) x_{(m+n) p^{i}}=(m, n) x_{(m+n) p^{i}} .
$$

It is clear that $(m, n) \equiv 0$ if and only if $m+n \geqq p$; hence

$$
y_{m}^{(i)} y_{n}^{(i)}= \begin{cases}(m, n) y_{m+n}^{(i)} & m+n<p, \\ 0 & m+n \geqq p .\end{cases}
$$

Thus (2.1) is satisfied; the remaining conditions (2.3), (2.4), and (2.5) are also satisfied as noted previously. Thus for each $i$ we have a subalgebra $A\left[y^{(i)}, p, t^{(i)}\right]$ (of binomial type). It remains to show that $T_{A}\left(x_{0}, x_{1}, x_{2}, \cdots\right)$ is isomorphic to their tensor product.

It follows from (2.6) and (2.7) that corresponding to the $p$-adic expansion (2.8) for $m$, we may write $x_{m}$ as a unique product

$$
x_{m}=x_{m_{0}} x_{m_{1} p} \cdots x_{m_{i} p^{i}}=y_{m_{0}}^{(0)} y_{m_{1}}^{(1)} \cdots y_{m_{i}}^{(i)} .
$$

Therefore the correspondence defined by

$$
\phi\left(x_{m}\right)=y_{m_{0}}^{(0)} \otimes y_{m_{1}}^{(1)} \otimes \cdots \otimes y_{m_{i}}^{(i)}
$$

establishes a module isomorphism (2.10). It remains to show that $\phi$ is multiplicative. Let (2.8) be the $p$-adic expansions of $m$ and $n$ (we may assume $i \leqq j)$. Then, using (2.6) and (2.7),

$$
\begin{aligned}
\phi\left(x_{m}\right) \phi\left(x_{n}\right) & =\left(y_{m_{0}}^{(0)} \otimes \cdots \otimes y_{m_{i}}^{(i)}\right)\left(y_{n_{0}}^{(0)} \otimes \cdots \otimes y_{n_{j}}^{(j)}\right) \\
& =\left(m_{0}, n_{0}\right) \cdots\left(m_{j}, n_{j}\right) y_{m_{0}+n_{0}}^{(0)} \otimes \cdots \otimes y_{m_{j}+n j}^{(j)} \\
& =(m, n) y_{m_{0}+n_{0}}^{(0)} \otimes \cdots \otimes y_{m_{j}+n_{j}}^{(j)}
\end{aligned}
$$

We consider 2 cases:

(i) If $(m, n) \equiv 0 \bmod p$ then $\phi\left(x_{m}\right) \phi\left(x_{n}\right)=0$. But also $\phi\left(x_{m} x_{n}\right)$ $=\phi(0)=0$.

(ii) If $(m, n) \not \equiv 0 \bmod p$ then none of the factors $\left(m_{r}, n_{r}\right), 0 \leqq r \leqq j$, are zero and hence $m_{r}+n_{r}<p$ for all $r$. But then

$$
\begin{aligned}
m+n & =\left(m_{0}+n_{0}\right)+\left(m_{1}+n_{1}\right) p+\cdots+\left(m_{j}+n_{j}\right) p^{j}, \\
\phi\left(x_{m+n}\right) & =y_{m_{0}+n_{0}}^{(0)} \otimes \cdots \otimes y_{m_{j}+n_{j}}^{(j)} .
\end{aligned}
$$

Thus (2.11) becomes

$$
\phi\left(x_{m}\right) \phi\left(x_{n}\right)=(m, n) \phi=\phi\left(x_{m+n}\right),
$$

completing the proof of Proposition 1.

\section{The main theorem.}

Theorem 1. Let $\left(E_{r}\right)$ be an initially decomposable acyclic canonical spectral sequence of $A$-algebras. If $B$ is a truncated polynomial algebra 
$A[x, h]$ where $x$ has even degree $m \geqq 2$, then

$$
F \cong \Lambda_{A}\left(x_{1}\right) \otimes T_{A}\left(z_{0}, z_{2}, z_{4}, \cdots\right)
$$

where $z_{1} \in F$ and is of degree $m-1$ and $z_{2 i} \in F$ and has degree $i(h m-2)$.

In view of Proposition 1 we have:

Corollary. (a) If in addition $A$ has characteristic zero then

$$
F \cong \Lambda_{A}\left(z_{1}\right) \otimes A\left[z_{2}, \infty, t\right],
$$

the second factor being of binomial type.

(b) If in addition $A$ has prime characteristic $p$ then

$$
F \cong \Lambda_{A}\left(z_{1}\right) \otimes_{i \geqq 0} A\left[z_{2 p^{i}}, p, t^{(i)}\right],
$$

each of the monogenic factors in the tensor product being of binomial type.

Proof. The following is a trivial consequence of (1.5):

$$
E_{r}^{p, q}=0 \quad \text { if } E_{2}^{p, 0}=0 \text { or } E_{2}^{0, q}=0
$$

We note further that the assumption on $B$ gives

$$
\begin{aligned}
& E_{2}^{p, 0}=0, \\
& \text { if } p \neq t m,(t=0,1, \cdots, h-1) \text {, } \\
& E_{2}^{t m, 0}=\left\{\begin{array}{l}
0 \\
A \cdot x^{t}
\end{array}\right. \\
& \text { if } t \geqq h \text {, } \\
& \text { if } 0 \leqq t<h \text {, }
\end{aligned}
$$

(by $A \cdot x^{t}$ we mean the free $A$-module generated by $x^{t}$ ). We shall now prove:

$$
\begin{aligned}
E_{2} & =E_{3}=\cdots=E_{m}, \\
E_{t m+1} & =E_{t m+2}=\cdots=E_{(t+1) m}, \quad t \geqq 1, \\
E_{(h-1) m+1} & =E_{(h-1) m+2}=\cdots=E_{\infty} .
\end{aligned}
$$

If $p \neq s m$ then, in view of (3.4) and (3.6), $E_{r}^{p, q}=0$ and hence $d_{r}\left(E_{r}^{p, q}\right)=0$. On the other hand, if $p=s m$ but $r$ is not a multiple of $m$ then

$$
d_{r}\left(E_{r}^{8 m, q}\right) \subset E_{r}^{8 m+r, q-r+1}=0
$$

(the latter module is zero by (3.4) and (3.6) since $s m+r$ is not a multiple of $m$ ). Thus $d_{r}=0$ if $r$ is not a multiple of $m$ and (3.7) and (3.7)' follow. If $r \geqq h m$ then by (3.4) and (3.6), $E_{r}^{p+r, q-r+1}=0$ and hence $d_{r}\left(E_{r}^{p, q}\right)=0$. Thus $E_{h m}=E_{h m+1}=\cdots=E_{\infty}$. Combining this with(3.7)' (taking $t=h-1$ ) gives $(3.7)^{\prime \prime}$. 
REMARK. Using these results it will be possible to identify $E_{2}^{0, q}$ $=E_{r}^{0, q}$ for some values of $q$ and $r(r>2)$. When we write $d_{r}(u)$, where $u \in E_{2}^{0, q}$, such an identification will be implied.

For convenience put $q_{0}=h m-2$. We shall now prove:

A. If $q \neq 0, m-1$, modulo $q_{0}$ then $E_{2}^{0, q}=0$.

B. There exists a sequence of generators $z_{0}=1, z_{1}, z_{2}, \cdots, z_{j}, \cdots$ for $F$ such that

$$
\begin{aligned}
E_{2}^{0, j g_{0}+(m-1)} & =A \cdot z_{2 j+1}, & d_{m}\left(z_{2 j+1}\right) & =x z_{2 j}, \\
E_{2}^{0, j q_{0}} & =A \cdot z_{2 j}, & d_{(h-1) m}\left(z_{2 j}\right) & =x^{h-1} z_{2 j-1},
\end{aligned}
$$

(note the preceding remark).

The proof is by induction on $q$. Let $\bar{q}>0$ and assume:

$\mathrm{A}_{\bar{q}}$. Statement $\mathrm{A}$ holds for all $q$ such that $0 \leqq q<\bar{q}$.

$\mathrm{B}_{\bar{q}}$. We have chosen generators $z_{0}=1, z_{2 j}\left(0<j q_{0}<\bar{q}\right)$, and $z_{2 j+1}$ $\left(m-1 \leqq j q_{0}+m-1<\bar{q}\right)$ such that (3.8) and (3.9) hold.

Clearly $A_{1}$ and $B_{1}$ are trivial; it remains to prove $A_{\bar{q}+1}$ and $B_{\bar{q}+1}$. We shall first prove

$$
E_{t m+1}^{0, \bar{q}}=E_{t m}^{0, \bar{q}}
$$

holds in the following cases:

(i) $\bar{q} \equiv 0\left(\bmod q_{0}\right), 1 \leqq t<h-1$.

(ii) $\bar{q} \equiv m-1\left(\bmod q_{0}\right), 1<t \leqq h-1$.

(iii) $\bar{q} \not \equiv 0, m-1\left(\bmod q_{0}\right), 1 \leqq t \leqq h-1$.

Consider

$$
0 \stackrel{d_{t m}}{\longrightarrow} E_{t m}^{0, \bar{q}} \stackrel{d_{t m}}{\longrightarrow} E_{t m}^{t m, \bar{q}-t m+1}
$$

Since $d_{t m} d_{t m}=0$, to prove (3.10) it suffices to show that the last module in (3.11) is zero. If $\vec{q}-t m+1<0$ this is trivial; hence assume $\bar{q}-t m+1 \geqq 0$. We may write $\bar{q}=j q_{0}+s, 0 \leqq s<q_{0}$.

(i) If $s=0$ and $1 \leqq t<h-1$ then $\bar{q}-t m+1=j q_{0}-t m+1 \neq 0$, $m-1\left(\bmod q_{0}\right)$. Therefore it follows from $\mathrm{A}_{\bar{q}}$ and (3.4) that $E_{t m}^{t m, \bar{q}-t m+1}$ $=0$.

(ii) $s=m-1$ and $1<t \leqq h-1$ then $\bar{q}-t m+1=j q_{0}+(1-t) m \neq 0$, $m-1\left(\bmod q_{0}\right)$. As in the preceding case we may conclude that $E_{t m}^{t m, \bar{q}-t m+1}=0$.

(iii) Suppose $s \neq 0, m-1$ and $1 \leqq t \leqq h-1$. We have $\bar{q}-t m+1$ $\equiv s-t m+1\left(\bmod q_{0}\right)$. If further $s \neq t m-1,(t+1) m-2$, then $\bar{q}-t m$ $+1 \not \equiv 0, m-1\left(\bmod q_{0}\right)$, and as in the preceding two cases we may conclude that the last module in (3.11) vanishes. It remains to consider the two exceptional cases:

If $s=t m-1$, then $\bar{q}-t m+1=j q_{0}$. Consider the map 


$$
d_{m}: E_{m}^{(t-1) m, j q_{0}+m-1} \rightarrow E_{m q}^{t m, j q_{0}} .
$$

Using (3.7) and (1.4) we have

$$
E_{m}^{t m, j q_{0}}=E_{2}^{t m, j q_{0}}=E_{2}^{t m, 0} \cdot E_{2}^{0, j q_{0}} .
$$

By hypothesis $E_{2}^{t m, 0}=A \cdot x^{t}$, and by $B_{\bar{q}}, E_{2}^{0, j q_{0}}=A \cdot z_{2 j}$. It follows from (3.13) that $E_{m}^{t m, j q_{0}}=A \cdot x^{t} z_{2 j}$. Similarly we may show $E_{m}^{(t-1) m, s q_{0}+m-1}$ $=A \cdot x^{t-1} z_{2 j+1}$. Now using the latter part of (3.8) we have

$$
d_{m}\left(x^{t-1} z_{2 j+1}\right)=d_{m}\left(x^{t-1}\right) z_{2 j+1}+x^{t-1} d_{m}\left(z_{2 j+1}\right)=x^{t} z_{2 j} .
$$

Thus (3.12) is an isomorphism and $E_{m+1}^{t m, j q_{0}}=0$. It follows that the last module in (3.11) is zero.

If $s=(t+1) m-2$, then $\bar{q}-t m+1=j q_{0}+m-1$. Since (3.12) is an isomorphism we have that $E_{m+1}^{(t-1) m, j g_{0}+m-1}=0$. The last module in (3.11) is therefore zero. This completes the proof of (3.10).

PROOF OF $A_{\bar{q}+1}$. Let $\bar{q} \not \equiv 0, m-1\left(\bmod q_{0}\right)$. Using $(3.7),(3.7)^{\prime},(3.7)^{\prime \prime}$, and (3.10) (case iii), we may write $E_{2}^{0, \bar{q}}=E_{\infty}^{0, \bar{z}}$ which is zero by acyclicity.

Proof of $B_{\bar{q}+1}$. We assert that the following maps are isomorphisms:

$$
\begin{array}{cc}
d_{m}: E_{m}^{0, \bar{q}} \rightarrow E_{m}^{m, \bar{q}-m+1}, & \text { if } \bar{q}=j_{q 0}+m-1 ; \\
d_{(h-1) m}: E_{(h-1) m}^{0, \bar{q}} \rightarrow E_{(h-1) m}^{(h-1) m, \bar{q}-(h-1) m+1} & \text { if } \bar{q}=j q_{0} .
\end{array}
$$

Assuming this we may prove $B_{i+1}$ as follows: Let $\bar{q}=j q_{0}+m-1$ : then $\bar{q}-m+1=j q_{0}<\bar{q}$. By (3.9), therefore $E_{2}^{0, j q_{0}}=A \cdot z_{2 j}$. Since also $E_{2}^{m, 0}=A \cdot x$, it follows from (1.4) that $E_{2}^{m, j q_{0}}=A \cdot x z_{2 j}$. Using (3.7) we may replace the subscript $m$ by 2 in each module in (3.14). If we therefore define $z_{2 j+1}=d_{m}^{-1}\left(x z_{2 j}\right)$ then (3.8) holds for $\mathrm{B}_{\bar{q}+1}$. Now let $\bar{q}=j q_{0}$. Then

$$
\bar{q}-(h-1) m+1=j q_{0}-(h-1) m+1=(j-1) q_{0}+m-1 .
$$

Since also $\bar{q}-(h-1) m+1<\bar{q}$, we have by $(3.8) E_{2}^{0, \bar{q}-(h-1) m+1}=A \cdot z_{2 j-1}$. By hypothesis, $E_{2}^{(h-1) m, 0}=A \cdot x^{h-1}$; hence it follows from (1.4) that

$$
E_{2}^{(h-1) m, \bar{q}-(h-1) m+1}=A \cdot x^{h-1} z_{2 j-1} .
$$

We assert that we may identify

$$
E_{(h-1) m}^{(h-1) m, q-(h-1) m+1}=E_{2}^{(h-1) m, \bar{q}-(h-1) m+1} .
$$

To prove this let $1 \leqq t<h-1$ and consider

$$
E_{t m}^{(h-1-t) m, 8} \stackrel{d_{t m}}{\longrightarrow} E_{t m}^{(h-1) m, \bar{q}-(h-1) m+1} \stackrel{d_{t m}}{\longrightarrow} E_{t m}^{(h-1+t) m, S}
$$


where $s$ and $S$ are the appropriate integers. The last module is evidently zero (by 3.4 ) since $h-1+t \geqq h$. Also

$$
s=q-(h-1) m+1+t m-1=(j-1) q_{0}+(t+1) m-2 .
$$

Evidently $0<(t+1) m-2<q_{0}$ and hence $s<\bar{q}$. Moreover, it is readily checked that $(t+1) m-2 \neq 0, m-1$. The first module in (3.18) is therefore zero in view of $A_{\bar{q}}$ and (3.4). It follows that

$$
E_{t m+1}^{(h-1) m, \bar{q}-(h-1) m+1}=E_{t m}^{(h-1) m, \bar{q}-(h-1) m+1}, \quad 1 \leqq t<h-1 .
$$

Using (3.7), (3.7)', and (3.19), the identification (3.17) then follows. We may also identify

$$
E_{2}^{0, j q_{0}}=E_{(h-1) m}^{0, j q_{0}}
$$

using (3.7), (3.7)', and (3.10) (case i). If we apply (3.17) and (3.20) in (3.15) and define $z_{2 j}=d_{m}^{-1}\left(x^{h-1} z_{2 j-1}\right)$ we see that (3.9) holds for $\mathrm{B}_{\bar{q}+1}$. It remains to prove that (3.14) and (3.15) are isomorphisms. Let $\bar{q}=j q_{0}+m-1$ and consider the sequence

$$
0 \stackrel{d_{m}}{\longrightarrow} E_{m}^{0, \bar{q}} \stackrel{d_{m}}{\longrightarrow} E_{m}^{m, j q_{0}} \stackrel{d_{m}}{\longrightarrow} E_{m}^{2 m, j q_{0}-m+1} .
$$

The last module is zero by (3.4) since by $\mathrm{A}_{\bar{q}}$ we have $E_{2}^{0, j q_{0}-m+1}=0$. Thus to prove (3.14) an isomorphism it suffices to show that $E_{m+1}^{0, \bar{q}}=0$ and $E_{m+1}^{m, y c_{0}}=0$. The former follows using $(3.7)^{\prime},(3.7)^{\prime \prime},(3.10)$ (case ii), and acyclicity. To prove the latter consider the sequence

$$
0 \stackrel{d_{t m}}{\longrightarrow} E_{t m}^{m, j q_{0}} \stackrel{d_{t m}}{\longrightarrow} E_{t m}^{(t+1) m, j q_{0}-t m+1}, \quad 1<t \leqq h-1 .
$$

If $t=h-1$ then $E_{2}^{(t+1) m, 0}=0$. If $1<t<h-1$ then $j q_{0}-t m+1 \neq 0, m-1$, modulo $q_{0}$, and hence $E_{2}^{0, j q_{0}-t m+1}=0$ by $\mathrm{A}_{\bar{q}}$. In either case the last module in (3.22) is therefore zero by (3.4) and hence $E_{t m}^{m, y q_{0}}=E_{t m+1}^{m, y q_{0}}$ for $1<t<h$. Combining this with $(3.7)^{\prime},(3.7)^{\prime \prime}$, and acyclicity it follows that $E_{m+1}^{m, j q_{0}}=0$. Finally, let $q=j q_{0}$ and consider the sequence

$$
\text { (3.23) } 0 \stackrel{d_{(h-1) m}}{\longrightarrow} E_{(h-1) m}^{0, j q_{0}} \stackrel{d_{(h-1) m}}{\longrightarrow} E_{(h-1) m}^{(h-1) m,(h-1) q_{0}+m-1} \stackrel{d_{(h-1) m}}{\longrightarrow} E_{(h-1) m}^{2(h-1) m,(j-1) q_{0}+h m} \text {. }
$$

The last module is evidently zero since $2(h-1) m>h m$. Moreover, $E_{(h-1) m+1}^{0, j q_{0}}=0, E_{(h-1) m+1}^{(h-1) m,(j-1) q_{0}+m-1}=0$ by $(3.7)^{\prime \prime}$ and acyclicity. Thus (3.23) reduces to the isomorphism (3.15). This completes the induction and $A$ and $B$ are proved.

Using $A$ and $B$ we shall now prove the following multiplication relations: 


$$
\begin{aligned}
z_{1}^{2} & =0, \\
z_{1} z_{2 j} & =z_{2 j+1}, \\
z_{2 i} z_{2 j} & =(i, j) z_{2(i+j)} .
\end{aligned}
$$

Note that $z_{1}^{2}$ has degree $2 m-2$ and $z_{2}$ has degree $h m-2$. Thus if $h>2, z_{1}^{2}=0$. If $h=2$ put $z_{1}^{2}=t z_{2}, t \in A$; then by (3.8) and (3.9) respectively, we have

$$
d_{m}\left(z_{1}^{2}\right)=x z_{1}-z_{1} x=x z_{1}-x z_{1}=0, \quad d_{m}\left(z_{1}^{2}\right)=t x z_{1} .
$$

Since $x z_{1}$ generates a free $A$-module, $t=0$ and (3.24) is established.

For $j=0,(3.24)$ is trivial. Let $j>0$ and assume (3.25) for all $j^{\prime}<j$. Put $z_{1} z_{2 j}=t z_{2 j+1},(t \in A)$; then using (3.8) we have

$$
d_{m}\left(z_{1} z_{2 j}\right)=x z_{2 j}-z_{1} d_{m}\left(z_{2 j}\right) .
$$

If $h=2$ then using (3.9), the inductive assumption, and (3.24) in succession,

$$
z_{1} d_{m}\left(z_{2 j}\right)=z_{1}\left(x z_{2 j-1}\right)=x z_{1} z_{1} x_{2 j-2}=0 .
$$

If $h>2$, note that $d_{m}\left(z_{2 j}\right) \in E_{2}^{m, j q_{0}-m+1}$ which is zero by (3.4) and A. In either case (3.27) reduces to $d_{m}\left(z_{1} z_{2 j}\right)=x z_{2 j}$. But by (3.8),

$$
d_{m}\left(z_{1} z_{2 j}\right)=t d_{m}\left(z_{2 j+1}\right)=t x z_{2 j} .
$$

Since $x z_{2 j}$ generates a free $A$-module, $t=1$ and (3.25) is proved.

For $i+j=0,(3.26)$ is trivial. Let $i+j>0$ and assume (3.26) for all $i^{\prime}+j^{\prime}<i+j$. If we put $z_{2 i} z_{2 j}=t_{i, j} z_{2(i+j)}$, then using (3.9),

$$
\begin{aligned}
\dot{a}_{(h-1) m}\left(z_{2 i} z_{2 j}\right) & =\left(x^{h-1} z_{2 i-1}\right) z_{2 j}+z_{2 i}\left(x^{h-1} z_{2 j-1}\right), \\
& =x^{h-1} z_{1}\left(z_{2 i-2} z_{2 j}+z_{2 i} z_{2 j-2}\right), \\
& =x^{h-1} z_{1}[(i-1, j)+(i, j-1)] z_{2 i+2 j-2}, \\
& =(i, j) x^{h-1} z_{2 i+2 j-1} .
\end{aligned}
$$

But also by (3.9),

$$
d_{(h-1) m}\left(z_{2 i} z_{2 j}\right)=t_{i, j} d_{(h-1) m}\left(z_{2(i+j)}\right)=t_{i, j} x^{h-1} z_{2(i+j)-1} .
$$

Since $x^{h-1} z_{2(i+j)-1}$ generates a free $A$-module, $t_{i, j}=(i, j)$.

The theorem now follows from (3.24), (3.25), and (3.26). For by (3.24), $z_{1}$ spans the subalgebra $\Lambda_{A}\left(z_{1}\right)$; by (3.26), the elements $z_{2 j}$ span the subalgebras $T_{A}\left(z_{0}, z_{2}, z_{4}, \cdots\right)$; and by (3.25), $F$ is clearly isomorphic to the tensor product of the two subalgebras under the obvious map.

4. Topological applications. Let $J$ denote a principal ideal domain of characteristic $p$ ( $p$ is then zero or a prime). 
Theorem 2. Let $X$ be a topological space whose singular cohomology algebra $H^{*}(X, J)=J[x, h]$ where $x$ is an element of even degree $m \geqq 2$. Let $\Omega$ denote the loop space of $X$ at a base point $x_{0}$.

(a) $H^{*}(\Omega, J) \cong \Lambda_{J}\left(z_{1}\right) \otimes J\left[z_{2}, \infty, t\right]$, if $p=0$, where $z_{1}$ and $z_{2}$ have degree $m-1$ and $h m-2$, respectively, and the second factor is of binomial type.

(b) $H^{*}(\Omega, J) \cong \Lambda_{J}\left(z_{1}\right) \otimes_{i \gtrless 0} J\left[z_{2 p i}, p, t^{(i)}\right]$, if $p$ is prime, where $z_{1}$ and $z_{2 p i}$ have degrees $m-1$ and $p^{i}(h m-2)$ respectively, and each $t^{(i)}$ is of binomial type.

Proof. Evidently $X$ is arcwise connected, simply connected, and torsion-free. Associated with the Serre fibering ${ }^{3} f: E \rightarrow X$ where $E$ is the space of paths beginning at $x_{0}$ (and $\Omega$ is the fibre at $x_{0}$ ) is a canonical spectral sequence of $J$-algebras $\left(E_{r}\right)$ which is acyclic (since $E$ is contractible) and such that $E_{2}^{p, q} \cong H^{p}(X, J) \otimes H^{q}(\Omega, J)$ (since $X$ is a torsion-free and $J$ is a principal ideal domain). These isomorphisms give an identification of the bigraded $J$-algebras, $E_{2}=H^{*}(X, J)$ $\otimes H^{*}(\Omega, J)$. Since the spectral sequence is initially decomposable, Theorem 2 follows immediately from Theorem 1 and its corollary.

Let $P_{n}$ and $Q_{n}$ denote the complex and quaternionic projective $n$ spaces, $1 \leqq n \leqq \infty$, respectively, and let $C$ denote the Cayley plane. Their cohomology algebras are known to be

$H^{*}\left(P_{n}, J\right)=J[x, n+1]$, degree of $x=2$;

$H^{*}\left(Q_{n}, J\right)=J[x, n+1]$, degree of $x=4$;

$H^{*}(C, J)=J[x, 3]$, degree of $x=8$.

Thus, Theorem 2 applies to $P_{n}, Q_{n}$, and $C$.

\section{REFERENCES}

1. A. Borel, Homology and cohomology of compact connected Lie groups, Proc. Nat. Acad. Sci. U.S.A. vol. 39 (1953) pp. 1142-1146.

2. J. P. Serre, Homologie singulière des espaces fibrés. Applications, Ann. of Math. vol. 54 (1951) pp. 425-505.

University of Chicago and

UNIVERSITY OF MICHIGAN

${ }^{8}$ See reference $[2$, Chapter IV]. 